\title{
Electrophysiological Effects of Lidocaine in Sick Sinus Syndrome
}

\author{
Yukiko Ishir, M.D., Hiroki Mrtsuda, M.D., Shin Eno, M.D., \\ Nobuyuki Fukui, M.D., Takashi Iwamoto, M.D., \\ Kazuhiro Fujitani, M.D., Yutaka Furuta, M.D., \\ Masao Yoshida, M.D., Akima Mryoshi, M.D., \\ Hironobu Tatershi, M.D., ${ }^{*}$ and Mutsuo Suzukawa, M.D.*
}

\section{SUMMARY}

Electrophysiological studies were performed to see the effects of lidocaine on the conduction system, particularly sinus node and atrium in 40 patients of SSS, using $\mathrm{HBE}$ recordings, rapid atrial pacing and atrial extrastimulus technique. Sinus cycle length, $\mathrm{PA}\left(\mathrm{P}^{\prime} \mathrm{A}\right), \mathrm{AH}, \mathrm{HV}$ intervals, calculated SACT, and refractory periods of atrium, AV node and His-Purkinje system did not change after lidocaine. Only maximum CSRT was significantly increased with lidocaine. These results were not affected by pretreatment of atropine.

In conclusion, the combining rapid atrial pacing with lidocaine may be useful to manifest the masked sinus node abnormalities. It was suggested that lidocaine directly depressed sinus node automaticity in SSS patients, without affecting perinodal tissue. Therefore, lidocaine should be used with caution in patients with known or suspected SSS.

\section{Additional Indexing Words :}

SRT SACT Refractory periods Atropine His bundle Electrogram

$\mathrm{L}$ IDOCAINE has been the most widely used for treatment of ventricular arrhythmias. The development of sinus arrest in some patients by lidocaine have been reported, ${ }^{1-8}$ ) but there were few reports concerning lidocaine effects on human sinus node and atrium. ${ }^{1,10)}$ In the present study, the electrophysiological effects of lidocaine on the conduction system, particularly sinus node and atrium, were studied in patients with sick sinus syndrome (SSS) by His bundle electrogram (HBE) recordings, rapid atrial pacing and atrial extrastimulus technique.

From the First Department of Internal Medicine, Hiroshima University School of Medicine, Hiroshima, Japan.

* The Department of Internal Medicine, Hiroshima City Hospital, Hiroshima.

Address for reprint: Yukiko Ishii, M.D., First Department of Internal Medicine, Hiroshima University School of Medicine, Kasumi 1-2-3-, Hiroshima, Japan.

Received for publication April 16, 1979. 


\section{Methons}

Patients: 40 patients with sinus node dysfunction aged 24 to 80 years (mean age 60.5 years, SSS group) and control group of 28 patients (mean age 46.5 years) were studied. The patients of SSS group were defined by the presence of one or more of the following abnormalities:11) 1) persistent unexplained sinus bradycardia (10 patients), 2) documented episodes of SA block and/or sinus arrest (19 patients), 3) bradycardia with episodic supraventricular tachyarrhythmias (11 patients). There were 16 men and 24 women. None had cardiac medication for at least 48 hours before the studies.

Electrophysiological studies: HBE were recorded using previously described method. ${ }^{12,13)}$ A quadripolar catheter was positioned at the high right atrium in the vicinity of sinus node for atrial pacing (distal 2 poles) and for recording high right atrial electrogram (proximal 2 poles). Signals from atrium and His bundle were recorded on a Mingograph at paper speeds of $100 \mathrm{~mm} / \mathrm{sec}$. Simultaneous electrocardiographic leads II and $V_{1}$ were also recorded. In all patients, $\mathrm{HBE}$ of spontaneous rhythm were recorded and atrial pacing were performed at 10 beats $/$ min increments in rate from $70 / \mathrm{min}$ to $180 / \mathrm{min}$. The PA(P'A), AH, HV intervals were measured at basic tracing and atrial pacing. Sinus node recovery time (SRT) was defined as the interval from the last paced $P$ wave to the first spontaneous $P$ wave after sudden cessation of the pacing for 15 or $60 \mathrm{sec}$ at various rates. The corrected SRT (CSRT) was defined as SRT minus the average sinus cycle length. Maximum SRT (max. SRT) and max. CSRT were defined as the longest SRT and CSRT, respectively. In 2 patients of SSS group, the recovery times were measured with the first spontaneous QRS deflection of ECG, because only junctional escape beats appeared after pacing. In 28 patients of SSS group and all patients of control group, refractory periods were measured using the atrial extrastimulus technique with the Fukuda-Denshi external pulse generator during sinus rhythm and atrial pacing with various cycle lengths $(850,700,600$, and/or $500 \mathrm{msec}$ ). Atrial premature beats were delivered with a variable coupling intervals after every 8 beats of the basic cycle at twice the diastolic threshold. Sinoatrial conduction time (SACT) was calculated by the following equation: SACT $=$ (return cycle-spontaneous sinus cycle) $\div 2$, (Zone of reset).

The following definition were used in regard to refractory periods: $S_{1}, A_{1}$, $H_{1}$, and $V_{1}$ represented the stimulus artifact, atrial, His bundle and ventricular electrograms of spontaneous sinus or driven beats, respectively. $\mathrm{S}_{2}, \mathrm{~A}_{2}, \mathrm{H}_{2}$, and $\mathrm{V}_{2}$ represented the stimulus artifact, atrial, His bundle, and ventricular electrograms in response to the extrastimulus, respectively. Effective refractory period (ERP) of the atrium (ERPA) was the longest $\mathrm{S}_{1}-\mathrm{S}_{2}$ interval, at which $\mathrm{S}_{2}$ did not propagate into the atrium. Functional refractory period (FRP) of the atrium (FRPA) was the shortest attainable propagated $A_{1}-A_{2}$ interval. ERP of the AV node (ERPAVN) was the longest $A_{1}-A_{2}$ interval, at which $A_{2}$ failed to depolarize the His bundle. FRP of the AV node (FRPAVN) was the shortest $\mathrm{H}_{1}-\mathrm{H}_{2}$ interval in response to any $A_{1}-A_{2}$. Relative refractory period of the His-Purkinje system (RRPhPs) was the longest $\mathrm{H}_{1}-\mathrm{H}_{2}$ interval at which $\mathrm{H}_{2}$ conducted to the ventricle with a longer HV interval and/or with an aberrant QRS complex.

Lidocaine was administrated intravenously in a dose of $1 \mathrm{mg} / \mathrm{Kg}$ after control 
recordings. The measurements were initiated $2 \mathrm{~min}$ after administration. Further, in 10 patients of SSS group, atropine was pre-medicated intravenously in a dose of $0.025 \mathrm{mg} / \mathrm{Kg}, 20 \mathrm{~min}$ before lidocaine. Max. CSRT were measured before and after lidocaine.

Statistical analysis of data was performed using Student's $t$ test for paired values, and the results were expressed as mean \pm standard errors of the mean.

\section{Results}

The mean sinus cycle length, the mean $\mathrm{PA}\left(\mathrm{P}^{\prime} \mathrm{A}\right), \mathrm{AH}$, and $\mathrm{HV}$ intervals during sinus rhythm and atrial pacing showed no significant difference between before and after lidocaine in both control and SSS groups (Table I). The HV interval remained fixed at all paced rates in each patient with normal $\mathrm{HV}$ interval. The HV interval increased in $2 / 3$ patients with initially prolonged $\mathrm{HV}$ interval ( $>55 \mathrm{msec}$ ) in SSS group. However, there were no significant difference between before and after lidocaine in $\mathrm{HV}$ interval of SSS group as a whole.

In control group, SRT and CSRT (at a rate of $130 / \mathrm{min}$ for $60 \mathrm{sec}$ ), max. SRT and max. CSRT did not change significantly with lidocaine. However, in SSS group, SRT, CSRT, max. SRT, and max. CSRT increased from 2,156 $\pm 282,994 \pm 269,2,935 \pm 317,1,769 \pm 302 \mathrm{msec}$ before to $2,882 \pm 370$, $1,627 \pm 342,3,472 \pm 398,2,317 \pm 384 \mathrm{msec}$ after lidocaine, respectively $(\mathrm{p}<$ $0.005, \mathrm{p}<0.005, \mathrm{p}<0.01$, and $\mathrm{p}<0.01$, Table I). The max. CSRT was

Table I. Electrophysiological Data before and after Lidocaine in Both Control and SSS groups

\begin{tabular}{l|r|r|r|r|r|r}
\hline & \multicolumn{3}{|c|}{ Control group } & \multicolumn{2}{c}{ Sick sinus syndrome group } \\
\cline { 2 - 7 } & Before & After & $\begin{array}{c}\mathrm{p} \\
\text { value }\end{array}$ & Before & After & p \\
value \\
\hline Sinus cycle length (S.C.L) & $871 \pm 34$ & $854 \pm 33$ & NS & $1184 \pm 54$ & $1158 \pm 54$ & NS \\
PA interval (S.C.L) & $31.2 \pm 1.6$ & $31.6 \pm 1.7$ & NS & $34.3 \pm 1.6$ & $36.3 \pm 1.5$ & NS \\
P'A interval (130/min A.P) & $49.9 \pm 3.3$ & $53.1 \pm 3.6$ & NS & $55.7 \pm 2.6$ & $56.3 \pm 3.1$ & NS \\
AH interval (S.C.L) & $93.4 \pm 2.9$ & $92.9 \pm 3.0$ & NS & $100.1 \pm 4.2$ & $99.7 \pm 4.6$ & NS \\
AH interval (130/min A.P) & $140.0 \pm 8.0$ & $145.1 \pm 8.3$ & NS & $170.4 \pm 10.1$ & $167.0 \pm 10.6$ & NS \\
HV interval (S.C.L) & $40.3 \pm 1.2$ & $41.1 \pm 1.3$ & NS & $42.2 \pm 1.2$ & $43.2 \pm 1.2$ & NS \\
HV interval (130/min A.P) & $40.9 \pm 1.6$ & $41.7 \pm 1.7$ & NS & $42.0 \pm 1.5$ & $42.1 \pm 1.6$ & NS \\
SRT (130/min 60 sec) & $1,030 \pm 42$ & $1,065 \pm 47$ & NS & $2,156 \pm 282$ & $2,882 \pm 370$ & $<0.005$ \\
CSRT (130/min 60 sec) & $185 \pm 25$ & $229 \pm 32$ & NS & $994 \pm 269$ & $1,627 \pm 342$ & $<0.005$ \\
Maximum SRT & $1,212 \pm 48$ & $1,191 \pm 48$ & NS & $2,935 \pm 317$ & $3,472 \pm 398$ & $<0.01$ \\
Maximum CSRT & $326 \pm 19$ & $326 \pm 24$ & NS & $1,769 \pm 302$ & $2,317 \pm 384$ & $<0.01$
\end{tabular}

Abbreviations: $A . P=$ atrial pacing; $S R T=$ sinus node recovery time; $C S R T=$ corrected sinus node recovery time.

Mean \pm S.E. (msec). 
abnormally prolonged ( $>525 \mathrm{msec}$ ) in $30 / 40(75 \%)$ patients of SSS group before lidocaine. In 5 of 10 patients who had normal max. CSRT before lidocaine, the max. CSRT increased toward abnormal value (>525 msec) after lidocaine. As a whole, the max. CSRT of SSS group was abnormally prolonged in $35 / 40(87.5 \%)$ patients after lidocaine. Further, in 10 patients of SSS group, the max. CSRT was prolonged to more than $1,000 \mathrm{msec}$ after lidocaine. Case S.Y. was a 71-year-old female with a history of syncope. The max. CSRT was 1,560 msec before, but markedly increased to over 6470 msec after lidocaine (Fig. 1). The effects of lidocaine on the max. CSRT were investigated in 10 patients of SSS group when the parasympathetic con-

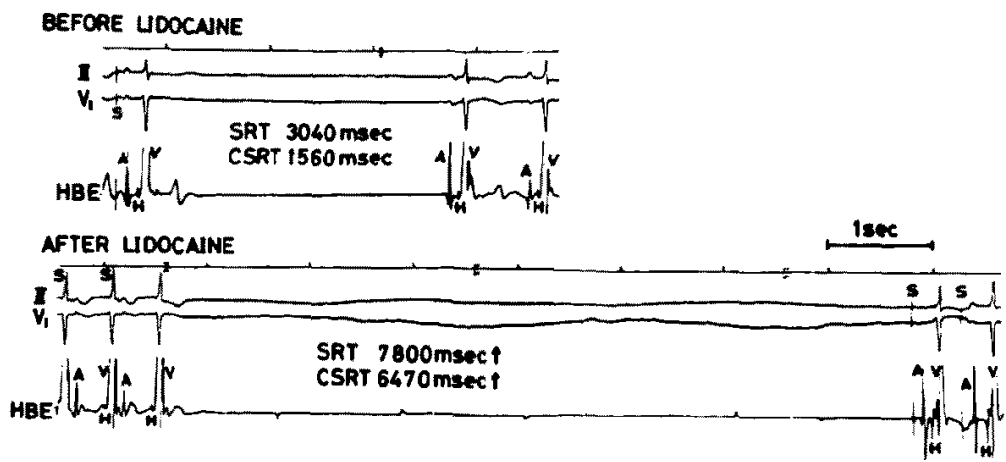

Fig. 1. Case S.Y. Typical example of remarkably increased maximum corrected sinus node recovery time ( $\max$. CSRT) after lidocaine. Upper panel represented before lidocaine with atrial pacing at a rate of $90 / \mathrm{min}$. Lower panel represented after lidocaine with atrial pacing at a rate of 130 / min. From top to bottom were ECG leads II, V1, and His bundle electrogram (HBE).

Table II. Effects of Lidocaine on Maximum CSR'T in SSS Patients Pretreated with Atropine (msec)

\begin{tabular}{c|c|c}
\hline Case No. & Before & After \\
\hline 1 & 1,440 & 2,740 \\
2 & 1,720 & 8,040 \\
3 & 260 & 260 \\
4 & 5,520 & 5,780 \\
5 & 360 & 305 \\
6 & 795 & 780 \\
7 & 830 & 780 \\
8 & 540 & 530 \\
9 & 2,140 & 4,890 \\
10 & 2,580 & 3,730 \\
\hline Mean & $1,619 \pm 498$ & $2,784 \pm 866$
\end{tabular}


Table III. Electrophysiological Data before and after Lidocaine in both groups

\begin{tabular}{l|c|c|c|c|c|c}
\hline & \multicolumn{3}{|c|}{ Control group } & \multicolumn{2}{c}{ Sick sinus syndrome group } \\
\cline { 6 - 7 } & Before & After & p value & Before & After & p value \\
\hline Calculated SACT & $98 \pm 6$ & $100 \pm 3$ & NS & $131 \pm 13$ & $139 \pm 13$ & NS \\
ERAA (S.C.L) & $261 \pm 6$ & $258 \pm 6$ & NS & $373 \pm 13$ & $366 \pm 14$ & NS \\
ERPA (Paired A.P) & $238 \pm 7$ & $231 \pm 4$ & NS & $294 \pm 7$ & $294 \pm 7$ & NS \\
FRPA (S.C.L) & $299 \pm 6$ & $292 \pm 6$ & NS & $413 \pm 12$ & $414 \pm 12$ & NS \\
FRPA (Paired A.P) & $282 \pm 4$ & $273 \pm 3$ & NS & $331 \pm 6$ & $333 \pm 5$ & NS \\
ERPAvN (S.C.L) & $316 \pm 12$ & $330 \pm 13$ & NS & $441 \pm 77$ & $466 \pm 33$ & NS \\
ERPAVN (Paired A.P) & $329 \pm 10$ & $327 \pm 9$ & NS & $386 \pm 14$ & $386 \pm 13$ & NS \\
FRPAVN (S.C.L) & $431 \pm 13$ & $444 \pm 12$ & NS & $501 \pm 70$ & $500 \pm 61$ & NS \\
FRPAVN (Paired A.P) & $436 \pm 10$ & $443 \pm 11$ & NS & $454 \pm 11$ & $472 \pm 12$ & NS \\
RRP PrPs (S.C.L) & $521 \pm 18$ & $482 \pm 18$ & NS & $559 \pm 30$ & $532 \pm 25$ & NS \\
RRPHPS (Paired A.P) & $418 \pm 18$ & $408 \pm 15$ & NS & $472 \pm 9$ & $467 \pm 19$ & NS
\end{tabular}

Abbreviations : $S A C T=$ sinoatrial conduction time ; $S C L=$ sinus cycle length ; $A . P=$ atrial pacing ; $E R P_{A}=$ atrial effective refractory period; $F R P_{A}=$ atrial functional refractory period; $E R P_{A V N}=$ AV node effective refractory period; FRPAVN $=A V$ node functional refractory period; RRPHPS = relative refractory period of the His-Purkinje system.

Mean \pm S.E. (msec).

trol of the heart had already been antagonized by atropine. The max. CSRT did not change in 6 patients but increased in 4 patients (Table II).

The calculated SACT was $98 \pm 6 \mathrm{msec}$ before and $100 \pm 3 \mathrm{msec}$ after lidocaine in control group (NS) and $131 \pm 13 \mathrm{msec}$ before and $139 \pm 13 \mathrm{msec}$ after lidocaine in SSS group (NS) (Table III). Before lidocaine, in 7 of 28 patients of SSS group, the SACT could not be calculated duc to showing a non-reset ( 2 patients) or a chaotic pattern ( 5 patients) of the return cycles. After lidocaine, the SACT could not be calculated in another 5 patients due to a chaotic pattern ( 3 patients) or ectopic atrial responses to atrial extrastimuli (2 patients). The calculated SACT was not decreased in any patient but increased by $25 \mathrm{msec}$ in only 4 patients of SSS group after lidocaine. However, in control group, there was no patient in whom the calculated SACT was prolonged.

ERPA (SCL, paired CL) and FRPA (SCL, paired CL) were not significantly changed with lidocaine in both groups. ERPAVN (SCL, paired GL), FRPAVN (SCL, paired CL), and RRPhPs (SCL, paired CL) were not significantly changed with lidocaine in both groups (Table III).

\section{Discussion}

Recently, several cases of severe sinus bradycardia and/or sinus arrest with lidocaine have been reported. ${ }^{1-8}$ ) In these cases, such arrhythmias 
were associated with or without preexistent sinus node dysfunction. However, the electrophysiological basis for these cases has not been fully elucidated. To clarify the mechanisms, electrophysiological studies of lidocaine effects on intracardiac conduction system were performed particularly in SSS patients. In the present study, lidocaine had no effect on sinus cycle length, $\mathrm{PA}\left(\mathrm{P}^{\prime} \mathrm{A}\right)$, $\mathrm{AH}$, and $\mathrm{HV}$ intervals, refractory periods of atrium, $\mathrm{AV}$ node, and $\mathrm{His}-$ Purkinje system and SACT in both groups. These observations were in agreement with previous ${ }^{9,101,15)-19 /}$ reports except sinus cycle length, RRPHPS and SACT.

Dhingra et $\mathrm{al}^{10}$ and Ryden et al ${ }^{14)}$ demonstrated that lidocaine significantly decreased sinus cycle length in patients both with and without sinus node dysfunction. They speculated the cause of decreased sinus cycle length might reflect the vagolysis and/or $\beta$-sympathetic stimulation (central). On the other hand, Roos et al ${ }^{91}$ demonstrated lidocaine had no significant effect on sinus cycle length in human with and without sinus node dysfunction, or Lieberman et $\mathrm{al}^{20)}$ and Mandel et al $^{21)}$ demonstrated negative chronotropic effects in animals with large doses $(5$ to $10 \mathrm{mg} / \mathrm{Kg}$ ). In the present study, the effects of lidocaine on sinus cycle length were varied inconsistently and not significant in both groups. These changes were thought to be due to subtle spontaneous variations in autonomic nervous control rather than the direct drug effect.

It was reported that lidocaine shortened RRPHPS in normal patients, ${ }^{19}$ ) and did not shorten but increased in patients with conduction disturbance in distal His bundle.9" In the present study, RRPHPs were not significantly changed in both groups.

Dhingra et $\mathrm{al}^{10}$ demonstrated lidocaine increased SACT and decreased SRT in patients with sinoatrial dysfunction but unchanged SACT and SRT in patients without. Decreased SRT could reflect increased sinus node automaticity and/or increased perinodal refractoriness, allowing entrance block to occur. While, Roos et al $^{91}$ demonstrated lidocaine did not change SRT in patients with and without sinus node dysfunction but they did not measure SACT. In experimental studies, there was no significant effect of lidocaine on SACT. ${ }^{22)}$ In this study, calculated SACT did not change after lidocaine in both groups in contrast to the Dhingra's observation. ${ }^{10)}$ The SRT, CSRT at a paced rate of $130 / \mathrm{min}$, max. SRT and max. CSRT increased significantly in SSS group and unchanged in control group after lidocaine. Even when vagal action was already abolished by atropine, max. CSRT was prolonged in 4/10 patients of SSS group. The major factors affecting SRT are sinus node automaticity, presence of entrance and/or exit block, and autonomic nervous control. It seemed difficult to consider the entrance and/or exit 
block from the results of unchanged SACT, ERPA, and FRPA. Furthermore, autonomic nervous control seemed not to play an important role from the results that atropine failed to protect the effects of lidocaine. Therefore, it was suggested that the most possible explanation of the increased SRT was due to depressed sinus node automaticity. The cause of this depression seen only in SSS group even with therapeutic dose was unknown but appeared that these patients might be more sensitive to lidocaine.

In SSS group, there were patients in whom max. CSRT remarkably increased or in some cases max. CSRT increased from normal before to abnormal value after lidocaine. Therefore, overdrive suppression test during stressing of the sinus node by lidocaine was useful to manifest the masked sinus node abnormalities. In addition, lidocaine should be used with caution in the treatment of ventricular arrhythmias of patients with known or suspected sinus node disease.

\section{REFERENCES}

1. Lippestad CT, Forfang K: Production of sinus arrest by lignocaine. Brit Med J 1: 537, 1971

2. Wood RA: Sinoatrial arrest. An interaction between phenytoin and lignocaine. Brit Med J 1: 645, 1971

3. Jeresaty $\mathrm{RM}, \mathrm{Kahn} A \mathrm{AH}$, Landry $\mathrm{AB}$ : Sinoatrial arrest due to lidocaine in a patient receving quinidine. Chest 61: 683, 1972

4. Cheng TO, Wadhwa K: Sinus standstill following intravenous lidocaine administration. JAMA 223: 790, 1973

5. Parameswaran R, Kahn D, Monhait R, Goldberg $\mathbf{H}$ : Sinus bradycardia due to lidocaine: clinical electrophysiologic correlations. J Electrocardiol 7: 75, 1974

6. Marriott HL, Phillips K: Profound hypotension and bradycardia after a single bolus of lidocaine. J Electrocardiol 7: 79, 1974

7. Levy S, Balansard P, Bouteau JM, Gerard R: L'arret Sinusal. Complication grave de la lidocaine intraveinuse. Arch Mal Coeur 68: 1069, 1975

8. Klein HO, Jutrin I, Kaplinsky E: Cerebral and cardiac toxicity of a small dose of lignocaine. Brit Heart J 37: 775, 1975

9. Roos JC, Dunning AJ: Effects of lidocaine on impulse formation and conduction defects in man. Am Heart J 89: 686, 1975

10. Dhingra RC, Deedwania PC, Cummings JM, Amat-Y-Leon F, Wu D, Denes P, Rosen KM: Electrophysiologic effects of lidocaine on sinus node and atrium in patients with and without sinoatrial dysfunction. Circulation 57: 448, 1978

11. Rubenstein JJ, Schulman CL, Yurchak PM, DeSanctis RW: Clinical spectrum of the sick sinus syndrome. Circulation $\mathbf{4 6}: 5,1972$

12. Ishii $\mathrm{Y}$, Iwamoto $\mathrm{T}$, Inomata $\mathrm{T}$, Kimura $\mathrm{K}$, Miyoshi $A$, Tateishi $H$, Suzukawa $M$ : Electrophysiological exmination in patients with sick sinus syndrome. The 1st report. J Hiroshima Med Ass 31 : 282, 1978

13. Ishii $Y$ : to be published

14. Ryden L, Cullhed I, Wasir H: Effect of lignocaine on heart rate in patients with sinus bradycardia associated with proven or suspected acute myocardial infarction. Cardiovasc Res 6: 664, 1972 
15. Bekheit S, Murtagh JG, Fletcher MP: Effect of lignocaine on conducting system of human heart. Brit Heart J 35: 305, 1973

16. Kunkel F, Rowland M, Scheinman MM: The electrophysiologic effects of lidocaine in patients with intraventricular conduction defects. Circulation 49: 894, 1974

17. Rosen MR, Merker C, Pippenger CE: The effects of lidocaine on the canine ECG and electrophysiologic properties of Purkinje fibers. Am Heart J 91 : 191, 1974

18. Gupta PK, Lichstein E, Chadda KD: Lidocaine-induced heart block in patients with bundle branch block. Am J Cardiol 33: 487, 1974

19. Josephson ME, Caracta AR, Lau SH: Effects of lidocaine on refractory periods in man. Am Heart J 84: 778, 1972

20. Lieberman NA, Harris RS, Katz RI, Lipschutz HM, Dolgin M, Fisher VJ: The effects of lidocaine on the electrical and mechanical activity of the heart. Am J Cardiol 22: 375, 1968

21. Mandel WJ, Bigger JT Jr: Electrophysiologic effects of lidocaine on isolated canine and rabbit atrial tissue. J Pharm Exp Therap 178: 81, 1971

22. Yamaguchi I, Mandel WJ: Studies on the effect of drugs on sinoatrial conduction. in The Conduction System of the Heart. Leiden, HE Stenfert Kroese BV, p 238, 1976 